\title{
Hubble Space Telescope Observations of Galilean Satellites
}

\author{
Melissa A. McGrath \\ Space Telescope Science Institute, 3700 San Martin Drive, Baltimore, \\ $M D$ 21218, USA
}

\begin{abstract}
One of the premier areas of scientific return from Hubble Space Telescope (HST) observations of Solar System objects has been studies of the Galilean satellites of Jupiter. Because these objects are unresolvable in most ground-based observations, HST's spatially resolved imaging and spectroscopy of their surfaces, atmospheres, and electrodynamic interactions with the Jovian magnetosphere have provided unique results. This talk will review highlights of the science results from HST observations of the Galilean satellites, including discovery of auroral emissions at the poles of Ganymede, the recent discovery of molecular sulfur in the Pele plume on Io, and the presence of $\mathrm{SO}_{2}$ in the surface of Callisto.
\end{abstract}

